\title{
Should a completion lymphadenectomy be performed after a positive biopsy of the sentinel node in melanoma? A vote for "no"
}

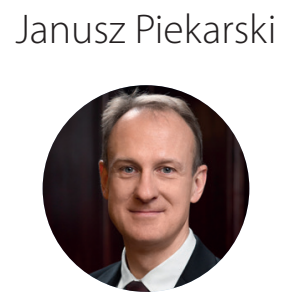

Clinic of Oncological Surgery, Medical University, Medical University of Lodz, Poland

Lymphadenectomy performed in patients with melanoma immediately after the detection of metastasis in the sentinel node has a real impact on the fate of only a few patients. Instead, it is a procedure that causes very severe adverse consequences. The question we have to ask ourselves is: In what number of patients do we cause complications by performing unnecessary procedures in order to save the few who need it?

In postoperative specimens after lymphadenectomy due to metastases in the sentinel node, metastases in non-sentinel lymph nodes are detected only in 18-30\% of cases [1-4]. It means that in $70-82 \%$ patients all melanoma lesions were removed during a previous biopsy of the sentinel node within the entire lymphatic flow. So it had been not only a diagnostic procedure, but also a therapeutic one. It means that in the vast majority of patients (even 80\%) lymphadenectomy was unnecessary from therapeutic point of view. Instead, it was burdened with a very high percentage of adverse consequences and complications. In the case of axillary lymphadenectomy, complications affect $50 \%$ procedures, and in the case of inguinal lymphadenectomy - as much as $90 \%$ [5-7]. These complications and adverse consequences are mainly: lymphorrhea, wound infections, sensory disturbances and lymphoedema. Especially lymphoedema is extremely troublesome and actually does not respond to treatment.

In about $80 \%$ patients with metastases to the sentinel node there is no need to remove lymph nodes. There is no doubt about that. This procedure is being performed because it is not yet clear which patients belong to these $80 \%$ and which do not.

This situation is not new in skin melanoma surgery. It has been discussed for decades whether regional lymph nodes should be excised immediately after the excision of the primary lesion in patients with skin melanoma of intermediate thickness or whether the excision should be delayed till clinically detectable metastases occur and only then should they be removed. The delay of lymphadenectomy was supported by the fact that lymph node metastases occurred only in a minority of patients and performing this procedure in all melanoma patients exposed most of them to unnecessary, but very burdensome, complications. On the other hand, lymphadenectomy was immediately supported by the conviction that if the procedure was performed quickly, the metastases from lymph nodes would be removed before they become a source of distant metastases. However, there was no method to indicate which patients had already developed lymph node metastases. Therefore, all patients were considered for lymphadenectomy. The solution to the problem was a sentinel node biopsy, a method that allowed for detecting clinically detectable metastases in lymph nodes. This made it possible to select those patients in whom lymphadenectomy was unjustified. However, in patients with identified metastases to the sentinel node, it was known that the process of lymphatic metastases had already begun and that lymphadenectomy was justifiable $[8,9]$.

As time went by and new clinical data were collected, it turned out that in most patients lymph node metastases occur only in the sentinel node. The excision of the sentinel node removes the whole cancer from the regional lymph nodes. Therefore, there is no point in removing the remaining lymph nodes. 
The problem, again decades later, is that the same thing boils down to questions: How to identify patients with metastases to non-sentinel nodes? Should lymphadenectomy be performed in all patients with metastases to the sentinel node or should we wait and perform lymphadenectomy only in those patients whose metastases in other lymph nodes are clinically manifested? If so, won't we make the prognosis worse for the patients? Clearly, we are waiting for a method that will allow us to identify those people who suffer from metastases to non-sentinel nodes. Such a method would save the majority of patients with metastasis to the sentinel node, unnecessary consequences and complications after the surgery.

However, another equally important question arises: Is it necessary to perform lymphadenectomy as soon as possible in those $20 \%$ patients with metastases in non-sentinel nodes?

There are two theories on solid cancer spreading. The first one, the "incubator" theory, is attributed to William Halsted. It dates back to the turn of the $19^{\text {th }}$ and $20^{\text {th }}$ centuries. According to it, the metastases from the primary lesion first get to the regional lymph nodes only through the lymphatic route. Cancer cells multiply within these lymph nodes but do not penetrate further. This happens only when the number of cancer cells reaches critical mass. This is when the hematogenous routes of metastatic spread to distant organs begins. According to this theory, if lymph nodes filled with metastases could be removed at the right time before the distant metastases began to develop, the further progress of the disease would be stopped and cured [10].

This theory was the reason why in the first half of the twentieth century the extent of surgical procedures escalated, believing that the more extensive the operation would be, the greater the chance that the cancer would be excised in its entirety and prevent the development of distant metastases. The best example were the procedures performed in patients with breast cancer. Although the extent of resection was even monstrous, this practice was effective only in some patients. In most cases, escalation of the resection extent proved to be ineffective. Patients were still dying from distant metastases and the degree of mutilation by surgery was unacceptable.

The second theory of the cancer spread is the so-called "marker"theory. According to it, metastases develop in parallel by hematogenous and lymphatic routes. The discovery of lymph node metastases only proves that cancer cells have a phenotype capable of metastasis and that the process has already begun [10].

There are many indications that in melanoma patients both scenarios are likely, but they occur in different proportions. Among melanoma patients, there are 5 basic groups [11]. The first two groups are patients with no metastases in regional lymph nodes. The larger of these two groups are those patients where cancer did not spread to lymph nodes or distant organs. In these patients, the biopsy of the sentinel lymph node brings true prognostic information, but the lymphadenectomy after the biopsy does not bring any benefits. The second group consists of patients with no metastases in regional lymph nodes, however, there were metastases to the distant organs through hematogenous route. In these patients, a negative biopsy of the sentinel node results in false negative prognostic information. Lymphadenectomy is not justified and does not change the fate of patients in whom distant metastases develop anyway.

In the other three groups there are people where metastases to regional lymph nodes occurred. The largest group are patients in whom both lymph node and distant metastases were formed very early, before the primary lesion resection. In these patients the biopsy of the sentinel node brings true prognostic information, but early lymphadenectomy - as soon as it is known that the sentinel node contains a metastasis does not change their fate, because they already have distant metastases, which determine their survival time.

The last two groups are people who, according to Halsted's theory, first develop lymph node metastases and only then develop distant ones. One group includes those in whom clinically undetectable lymph node metastases start to function as a source of distant metastases after reaching the size of clinically detectable metastases. In such cases, the biopsy of the sentinel node brings true prognostic information. Early lymphadenectomy immediately after confirmation of metastasis in the sentinel node is not justified, as delaying it till detectable metastases manifest is safe. However, late lymphadenectomy and removal of cancer from the regional lymphatic flow prevent the development of distant metastases and end the course of the disease

The last group and the only one in which early lymphadenectomy is justified are patients with regional lymph node metastases, which may be a source of distant metastases already at the stage of clinically detectable metastases (detected only by biopsy of a sentinel node). In such patients, a sentinel node biopsy provides true prognostic information and early lymphadenectomy may be crucial for survival. However, this is a very small group of patients with melanoma [11]. If we add to this the fact that most of these people have metastases in sentinel nodes and all melanoma lesions are removed during a sentinel node biopsy, then the group in which early supplemental lymphadenectomy is justified turns out to be even smaller.

The reasoning presented above turns out to be confirmed by the research carried out so far. Early lymphadenectomy did not affect overall survival in any of the randomized trials performed in patients with melanoma with no clinically detectable lymph node metastases. This applies both before and after the introduction of a sentinel node biopsy [11]. These observations were the reason for two randomized clinical trials aimed at assessing the impact of early lymphadenectomy on the survival of patients with metastases to sentinel nodes.

The first one, the DeCOG-SLT study, was a multi-center, randomized, third phase study in which patients with torso 
and limb melanoma were included and treated in 41 centers in Germany [12]. Patients with metastases in sentinel nodes were randomly assigned 1:1 to 2 arms of the study. Lymphadenectomy was performed on one arm in the case of the second arm the patients were carefully monitored with a regional lymph node ultrasound every 3 months. In this arm lymphadenectomy was performed only in patients in whom regional lymph node metastases were clinically manifested. The first priority end point of the trial was the time of survival without distant metastases. Ultimately, 483 patients agreed to participate in the study. After three years of follow-up, 74.9\% of patients undergoing lymphadenectomy and $77.0 \%$ of patients undergoing observation had survived without distant metastases. The difference was statistically insignificant $(p=0.87)$. The results of the total survival analysis were similar. In the group of patients undergoing lymphadenectomy, $81.2 \%$ of patients were still alive after 3 years, which referred to $81.7 \%$ of patients in the group of patients under observation. The difference was also not statistically significant.

The second study, MSLT-II, was a multi-center, international study of the third phase, in which 63 centers were involved [13]. In total, 1934 patients were analyzed for survival time till death due to melanoma. As in the previous study, patients with sentinel node metastases were randomly assigned to a group where an immediate completion lymphadenectomy was performed or to a group where they were observed, and lymphadenectomy was performed only when the regional lymph node metastases were clinically manifested. The ultrasound examination of regional lymphatic flow was performed every 4-6 months. The percentage of alive patients (melanoma-specific survival) after 3 years in both groups was the same and amounted to $86.1 \%$. The researchers found that the percentage of relapse-free survival was higher in the group undergoing immediate lymphadenectomy than in the group under observation, but it was caused by relapses within the unremoved regional lymph nodes and not by distant metastases. The difference in relapse-free survival was at the statistical significance limit $(p=0.05)$ and did not translate into the percentage of melanoma-specific survival. The researchers concluded that immediate lymphadenectomy after the diagnosis of metastasis in the sentinel node improved the indicators of regional disease control and provided prognosis information, but did not improve melanoma-specific survival.

The results of both randomized studies cited above aroused great controversy. Opponents point to numerous limitations in the usefulness of their results, which are mainly due to the fact that the time taken to evaluate survival is too short. Therefore, at the end it is worth to quote the results of the latest meta-analysis published in April 2019 in the Journal of Surgical Research [4]. The researchers analyzed the fate of 7966 patients who participated in 12 studies. Calculations showed that immediate regional lymphadenectomy performed after detecting metastasis in the sentinel node significantly impro- ved the percentage of progression-free survival after 3 years (71.0\% compared to $66.2 \%$ in the observation group; $p=0.02$ ) and after 5 years (48.3\% compared to $47.8 \% ; p=0.02$ ). However, there was no difference in melanoma-specific survival after both 3 and 5 years (5-year survival: 68.4\% compared to 69.8\% in the observation group; $p=0.78$ ), as well as no difference in total survival after 5 years (68.2\% compared to $78.9 \%$ in the observation group; $p=0.78$ ). The authors concluded that immediate regional lymphadenectomy in patients with sentinel node metastasis significantly improved the percentage of disease-free survival after 3 and 5 years, but it did not translate into improved melanoma-specific or total survival [4].

Summarizing the presented trial results, it seems that the answer to the question: "Is it justified to perform lymphadenectomy in patients with metastases in the sentinel node?" is: No. However, this answer must be subject to at least two reservations.

First, the results were obtained under specific conditions created by the clinical trials. Reproducing these conditions in reality is not always possible. In clinical trials, patients under observation were regularly subject to ultrasound examinations in order to detect a clinically detectable disease as early as possible. If performing a regular ultrasound surveillance is not possible, it may not be safe to give up lymphadenectomy in favor of observation. The patient should then be qualified for lymphadenectomy.

Secondly, lymphadenectomy should be performed when there are other factors that may cause lymphadenopathy in patients. Especially that it can effectively mask the relapse of the disease despite careful ultrasound surveillance.

\section{Conflict of interest: none declared}

Janusz Piekarski

Medical University of Lodz, Poland

Clinic of Oncological Surgery

ul. Pomorska 251

92-213 Łódź, Poland

e-mail:Janusz.piekarski@umed.lodz.pl

\section{Received and accepted: 14 May 2019}

\section{References}

1. Schuitevoerder D, Bubic I, Fortino J et al. Patients with sentinel lymph node melanoma: who needs completion lymph node dissection? Am J Surg 2018; 215: 868-872.

2. Gershenwald JE, Andtbacka RH, Prieto VG et al. Microscopic tumor burden in sentinel node involvement in patients with melanoma. $J$ Clin Oncol 2008; 26: 4296-4303.

3. Pasquali $\mathrm{S}$, Mocellin S, Mozzillo $\mathrm{N}$ et al. Nonsentinel lymph node status in patients with cutaneous melanoma: results from multi-institution prognostic study. J Clin Oncol 2014; 32: 935-941.

4. Macedo Fl, Fayne RA, Azab B et al. The role of completion lymphadenectomy in positive regional lymph nodes in melanoma: a meta analysis. J Surg Res 2019; 236: 83-91.

5. Guggenheim MM, Hug U, Jung FJ et al. Morbidity and recurrence after completion lymph node dissection following sentinel lymph node biopsy in cutaneous malignant melanoma. Ann Surg 2008; 247: 687-693.

6. Starz H, Balda BR, Kramer Kl et al. A micromorphometry-based concept for routine classification of sentinel lymph node metastases and its clinical relevance for patients with melanoma. Cancer 2001; 91: 2110-2112. 
7. Pasquali S, Spillane AJ, de Wilt JH et al. Surgeon's opinions on lymphadenectomy in melanoma patients with positive sentinel nodes: a worldwide web-based survey. Ann Surg Oncol 2012; 19: 4322-4329.

8. Morton DL, Thompson JF, Essner R et al. Validation of the accuracy of intraoperative lymphatic mapping and sentinel lymphadenectomy for early -stage melanoma: a multicenter trial. Multicenter Selective Lymphadenectomy Trial Group. Ann Surg 1999; 230: 453-463.

9. Morton DL, Cochran AJ. The case for lymphatic mapping and sentinel lymphadenectomy in the management of primary melanoma. $\mathrm{Br} J$ Dermatol 2004; 151: 308-319.
10. Faries MB. Completing the dissection in melanoma: increasing decision precision. Ann Surg Oncol 2018; 25: 585-587.

11. Bartlett EK. Current management of regional lymph nodes in patients with melanoma. J Surg Oncol 2019; 119: 200-207.

12. Leiter U, Stader R, Mauch $C$ et al. Complete lymph node dissection versus no dissection in patients with sentinel lymph node biopsy positive melanoma (DeCOG-SLT): a multicentre, randomised, phase 3 trial. Lancet Oncol 2016; 17: 757-767.

13. Faries MB, Thompson JF, Cochran AJ et al. Completion dissection or observation for sentinel-node metastasis in melanoma. $N$ Engl J Med $2017 ; 376 ; 2211-2222$. 